\title{
A new name for Pterospermum gracile P. Wilkie (Malavaceae: Dombeyoideae)
}

\section{R. Kottaimuthu ${ }^{1}$}

Summary. A new name, Pterospermum peterianum is proposed as a replacement name for $P$. gracile $\mathrm{P}$. Wilkie as it is an illegitimate later homonym of $P$. gracile Geyl.

Key Words. Homonym, nomen novum, Thailand.

\section{Introduction}

The genus Pterospermum Schreb. belongs to the subfamily Dombeyoideae of Malvaceae (Bayer \& Kubitzki 2003; Wilkie et al. 2006). It is comprised of 25 - 30 species and is distributed from the eastern Himalayas and southern China, through South-East Asia to the Moluccas in tropical Asia (Wilkie 2007).

After the publication of Sterculiaceae of Thailand (Phengklai 2001) a new species Pterospermum gracile was described by Peter Wilkie in 2013. However, according to Article 53.1 of the ICN (McNeill et al. 2012) it is an illegitimate later homonym of a fossil taxon Pterospermum gracile Geyl. (Geyler 1877: 80). Hence a new name is proposed here for this later homonym.

\section{Nomenclature}

Pterospermum peterianum Kottaim., nom. nov.

http://www.ipni.org/urn:lsid:ipni.org:names:60476581-2

Replaced synonym: Pterospermum gracile P. Wilkie, Thai Forest Bull., Bot. 41: 10 (2013), nom. illeg. non Pterospermum gracile Geyl., Palaeontographica, Suppl. 3 (1): 80 (Geyler 1877). Type: Thailand, Krabi Province, Muang Krabi Distr., Khao Phanom Bencha National Park, Headquarters, $8^{\circ} 14^{\prime} \mathrm{N}$, $98^{\circ} 55^{\prime} \mathrm{E}$, rocky semi-open area next to permanent stream at edge of lowland evergreen forest, S. Gardner E $P$. Sidisunthorn ST2171 (holotype E; isotypes $\mathrm{BKF}, \mathrm{K})$.

DISTRIBUTION. Peninsular Thailand. ETYMOLOGY. Named after Dr Peter Wilkie, Royal Botanic Garden, Edinburgh.

\section{Acknowledgements}

I am grateful to the Management, Thiagarajar College, Madurai for facilities and encouragement. I also wish to thank Dr D. Kannan, Head, Department of Botany for constant support.

\section{References}

Bayer, C. \& Kubitzki, K. (2003). Malvaceae. In: K. Kubitzki \& C. Bayer (eds), The Families and Genera of Vascular Plants, Vol. 5: 225 - 311. Springer Verlag, Berlin.

Geyler, H. T. (1877). Ueber fossile Pflanzen von Borneo. Palaeontographica, Suppl. Bd. 3: 60 - 84.

McNeill, J., Barrie, F. R., Buck, W. R., Demoulin, V., Greuter, W., Hawksworth, D. L., Herendeen, P. S., Knapp, S., Marhold, K., Prado, J., Prud'homme van Reine, W. F., Smith, G. F., Wiersema, J. H. \& Turland, N. J. (eds) (2012). International code of nomenclature for algae, fungi, and plants (Melbourne Code): Adopted by the eighteenth International Botanical Congress, Melbourne, Australia, July 2011. Regnum Veg. 154: 1 - 274.

Phengklai, C. (2001). Sterculiaceae. In: T. Santisuk \& K. Larsen (eds), Flora of Thailand 7 (3): 539 - 654. The Forest Herbarium, Bangkok.

Wilkie, P. (2007). A new species of Pterospermum (Dombeyoideae, Malvaceae/ Sterculiaceae) from Cambodia and Vietnam. Edinburgh J. Bot. 64(2): 179 - 183.

(2013). A new species of Pterospermum Schreb. (Dombeyoideae, Malvaceae/ Sterculiaceae) from southern Thailand. Thai Forest Bull., Bot. 41: 10 - 12.

Clark, A., Pennington, R. T., Cheek, M., Bayer, C. \& Wilcock, C. C. (2006). Phylogenetic relationships within the subfamily Sterculioideae (Malvaceae/Sterculiaceae-Sterculieae) using the chloroplast gene ndhF. Syst. Bot. 31: $160-170$.

Accepted for publication 19 June 2018. Published online 25 July 2018

1 Department of Botany, Thiagarajar College, Madurai-625009, Tamil Nadu, India. e-mail: kottaimuthu@yahoo.co.in 